\title{
Results from the SMART-1 lunar mission
}

\author{
Bernhard H. Foing \\ Space Science Department, EsTec, ESA, Noordwijk, the Netherlands \\ email: bernard.foing@esa.int
}

Abstract. Results of the SMART-1 mission to Earth's Moon are summarized.

Keywords. Moon, space vehicles

\section{Results}

SMART-1 is the first ESA mission that reached the Moon. It demonstrated Solar Electric Primary Propulsion (SEP) and tested new technologies for spacecraft and instruments.

Launched on 27 Sept. 2003, as Ariane-5 auxiliary passenger, SMART-1 spiraled out towards lunar capture on 15 November 2004, and then towards lunar science orbit, which was reached on 1 March 2005. The mission has been extended, to end with an impact on 2-3 September 2006.

This mission's purpose is not only to perform science but also to prepare future international lunar exploration, in collaboration with upcoming missions.

The $19 \mathrm{~kg}$ payload includes a miniaturized high-resolution camera AMIE, a near-infrared point-spectrometer sIR for mineralogy investigation, and a very compact X-ray spectrometer D-CIXS for surface elemental composition. There is also an experiment (KaTE) aimed at demonstrating deep-space telemetry and telecommand communications in the $\mathrm{X}$ and Ka-bands, a radio-science experiment RSIS, a deep space optical link (Laser-Link Experiment), using the ESA Optical Ground station in Tenerife, and the validation of a system of autonomous navigation (OBAN) based on image processing.

SMART-1 lunar science investigations include studies of the chemical composition of the Moon, of geophysical processes (volcanism, tectonics, cratering, erosion, deposition of ices and volatiles) for comparative planetology, and high resolution studies in preparation for future steps of lunar exploration. The mission addresses several topics such as the accretional processes that led to the formation of rocky planets, and the origin and evolution of the Earth-Moon system.

\section{Mission details}

Launch date: 27 September 2003

Arrival date: 15 November 2004

Payload mass: $19 \mathrm{~kg}$.

Primary science instruments:

$S M A R T$-1-AMIE camera: surface images in visible and near-infrared light.

$S M A R T$-1-SIR infrared spectrometer: maps lunar minerals.

$S M A R T$-1-D-CIXS X-ray spectrometer: identifies key chemical elements in the lunar surface. 
SMART-1-XSM X-ray detector: monitors variations in solar X-ray emissions, studies solar variability.

SMART-1-SPEDE electric field experiment: monitors the Moon's wake in the solar wind. $S M A R T$-1-RSIS radio science experiment: measures lunar librations.

\section{In the literature}

A detailed description of the SMART-1 mission plan was published in the January 1999 issue of Earth, Moon, and Planets. Racca et al. (1999) outline the mission itself, while Foing et al. (1999) discusses the science goals of the mission.

The first results from the mission were published in Advances in Space Research in January 2006. Foing et al. (2006) describe the status, first results and goals of the SMART-1 mission, while Josset et al. (2006) describe the science objectives and first results from the $S M A R T-1 / \mathrm{AMIE}$ multicolour micro-camera.

\section{References}

Foing, B. H., Heather, D. J., Almeida, M., \& the SMART-1 Science Technology Working Team 1999, Earth, Moon, and Planets, 85-86, 379

Foing, B. H., Racca, G. D., Marini, A., Evrard, E., Stagnaro, L., Almeida, M., Koschny, D., Frew, D., Zender, J., Heather, J., Grande, M., Huovelin, J., Keller, H. U., Nathues, A., Josset, J. L., Malkki, A., Schmidt, W., Noci, G., Birkl, R., Iess, L., Sodnik, Z., \& McManamon, P. 2006, Adv. Sp. Res., 37, 6

Josset, J.-L., Beauvivre, S., Cerroni, P., de Sanctis, M. C., Pinet, P., Chevrel, S., Langevin, Y., Barucci, M. A., Plancke, P., Koschny, D., Almeida, M., Sodnik, Z., Mancuso, S., Hofmann, B. A., Muinonen, K., Shevchenko, V., Shkuratov, Yu., Ehrenfreund, P., \& Foing, B. H. 2006, Adv. Sp. Res., 37, 14

Racca, G. D., Foing, B. H., \& Coradini M. 1999, Earth, Moon, and Planets, 85-86, 379 